\title{
Joint Radiomics Analysis on 18F-FDG-PET-MRI of Primary Tumor to Predict the Risk of Synchronous Distant Metastasis in Pancreatic Ductal Adenocarcinoma
}

\section{Jing Gao}

Shanghai Jiao Tong University Medical School Affiliated Ruijin Hospital

\section{Yaya Bai}

Shanghai Jiao Tong University Medical School Affiliated Ruijin Hospital

Fei Miao

Shanghai Jiao Tong University Medical School Affiliated Ruijin Hospital

\section{Xinyun Huang}

Shanghai Jiao Tong University Medical School Affiliated Ruijin Hospital

\section{Markus Schwaiger}

Klinikum rechts der Isar der Technischen Universität München: Klinikum rechts der Isar der Technischen Universitat Munchen

\section{Axel Rominger}

Inselspital University Hospital Bern: Inselspital Universitatsspital Bern

\section{Biao Li}

Shanghai Jiao Tong University Medical School Affiliated Ruijin Hospital

\section{Hui Zhu}

Shanghai Medical University: Fudan University

xiaozhu Lin ( $\nabla$ lxz11357@rjh.com.cn )

Shanghai Jiao Tong University School of Medicine https://orcid.org/0000-0002-8195-3932

\section{Kuangyu Shi}

Munich University of Technology: Technische Universitat Munchen

\section{Research Article}

Keywords: Pancreatic Ductal Adenocarcinoma, Metastasis, Radiomics, MRI, 18F-FDG-PET

Posted Date: August 13th, 2021

DOI: https://doi.org/10.21203/rs.3.rs-801341/v1 
License: (c) (i) This work is licensed under a Creative Commons Attribution 4.0 International License. Read Full License 


\section{Title:Joint radiomics analysis on ${ }^{18}$ F-FDG-PET-MRI of primary tumor to predict the risk of synchronous distant metastasis in pancreatic ductal adenocarcinoma}

Jing $\mathrm{Gao}^{1}$, Yaya $\mathrm{Bai}^{1}$, Fei $\mathrm{Miao}^{2}$,Xinyun Huang ${ }^{1}$, Markus Schwaiger ${ }^{3}$, Axel Rominger ${ }^{4}$, Biao $\mathrm{Li}^{1}$, Hui Zhu ${ }^{6 *}$,Xiaozhu Lin ${ }^{1 *}$, Kuangyu Shi ${ }^{4,5}$

1 Department of Nuclear Medicine, Ruijin Hospital, Shanghai Jiao Tong University School of Medicine, Shanghai, China

2 Department of Radiology, Ruijin Hospital, Shanghai Jiao Tong University School of Medicine, Shanghai, China

3 Klinikum rechts der Isar, Technical University of Munich, Munich, Germany

4 Department of Nuclear Medicine, University of Bern, Switzerland.

5 Department of Informatics, Technical University of Munich, Germany

6 Department of Diagnostic Radiology, Fudan University Shanghai Cancer Center; Department of Oncology, Shanghai Medical College, Fudan University Jing Gao and Yaya Bai contributed equally to this work.

*corresponding author:

Xiaozhu Lin

Department of Nuclear Medicine, Ruijin Hospital, Shanghai Jiao Tong University School of Medicine, No. 197 Ruijin Er Road, Shanghai 200025, China 1xz11357@rjh.com.cn

Hui Zhu

Department of Diagnostic Radiology, Fudan University Shanghai Cancer Center; Department of Oncology, Shanghai Medical College, Fudan University; 270 DongAn Road, Shanghai, 200032, China 
huizhu171717@163.com 


\section{Abstract}

Purpose: This study explores the potential of joint radiomics analysis of PET-MRI of primary pancreatic ductal adenocarcinoma (PDAC) tumor in predicting the risk of synchronous distant metastasis (SDM).

Methods: Two cohorts of PDAC patients were collected including a development cohort $(\mathrm{n}=66)$ receiving separate ${ }^{18}$ F-FDG-PET/CT and multi-sequence MRI, and a external test cohort $(n=25)$ receiving hybrid PET/MR. All of these patients were confirmed with SDM after imaging. Radiomics features of primary PDAC tumors were selected and models were built for PET, MRI, and PET-MRI from the development cohort. A radiomics nomogram was constructed by combining independent clinical indicators. The developed radiomics nomogram was independently evaluated on the test cohort.

Results: The area under the curve (AUC) values of PET, MRI, and joint PET-MRI models were $0.89,0.86$, and 0.94 in the training set and $0.77,0.67$, and 0.77 in the test set. The radiomics nomogram combing the joint PET-MRI radiomics signature, age, and CA19-9 level had good calibration and high discrimination capacity with maximum AUC value (0.93). The decision curve analysis (DCA) confirmed the radiomics nomogram had clinical usefulness. The evaluation on the independent test cohort showed that the accuracy, sensitivity, specificityand AUC values of radiomics nomogram were $84.0 \%, 78.6 \%, 90.9 \%$ and 0.85 .

Conclusion: The robust and effective prediction of the risk of SDM for the preoperative PDAC patients confirmed the potential of the radiomics analysis on 
PET/MR. The radiomics information in primary tumor may provide complementary and alerting hints for cancer staging.

\section{Keywords}

Pancreatic Ductal Adenocarcinoma, Metastasis, Radiomics,MRI, ${ }^{18}$ F-FDG-PET. 


\section{Introduction}

Pancreatic ductal adenocarcinoma (PDAC)is characterized by late diagnosis, high mortality rate, and poor prognosis $[1,2]$. Approximately $50 \%$ of new PDAC cases were detected with distant metastases at diagnosis of the primary tumor[3], which would preclude a radical therapeutic approach and indicate poor prognosis[4]. And most patients undergoing surgical resection will have metastasis within 4 years after surgery, which indicates that micrometastasis actually exists in patients with obviously limited tumors[5]. The treatment options available for patients with pancreatic cancer depend on the primary disease stage. Patients are eligible for curative surgery for nonmetastatic tumors and metastatic tumors may benefit from palliative chemotherapy. PDAC is characterized by a higher heterogeneous risk of metastasis[6]. Therefore, the detection of distant metastases is imperative for sparing from unnecessary pancreatectomy and selecting appropriate treatment strategies in PDAC patients.In addition, neoadjuvant treatment is the accepted approach for resectable patients with high risk features according to 2021 NCCN guidelines[7].

${ }^{18} \mathrm{~F}$-fluorodeoxyglucose (FDG) positron emission tomography/computed tomography (PET/CT) is a diagnostic tool in detecting distant metastases[8, 9].However, it is suboptimal in the evaluation of liver lesions due to high background hepatic FDG uptake[10] and the detection capability of subtle peritoneal carcinomatosis in PDAC is still limited[11]. Compared with CT, multi-phase contrastenhanced (CE) sequences and diffusion-weighted imaging (DWI) sequences of MRI are useful for the presence of liver metastases[12, 13] and peritoneal metastases[14] 
from PDAC. Moreover, FDG-PET and MRI can provide both functional metabolic information from PET and better tissue contrast and anatomical resolution from MRI. However, FDG-PET might miss liver/peritoneal metastatic tumors that were $<1 \mathrm{~cm}[15]$ and MRI had limitations in detecting liver metastasis smaller than $3 \mathrm{~mm}[16]$ and lung metastasis. Therefore, a new tool that could improve the sensitivity and accuracy of imaging techniques are needed.

Radiomics converted digital medical images into high-throughput quantitative features for clinical decision support and improved diagnostic accuracy[17]. Tumor intensity, shape, and textural features extracted from radiomics are thought to have the potential to reflect intratumoral histopathological properties[18].A recent study demonstrated the use of nomograms based on clinicopathological features for predicting liver metastasis in 12644 patients with PDAC[19].However,pathologic information was obtained by examining the surgical specimen and thus cannot be applied to guide therapeutic strategies. Accordingly, preoperative noninvasive biomarkers are needed to predict the occurrence of synchronous distant metastasis (SDM). ${ }^{18}$ F-FDG-PET and CE-MRI offer the opportunity to study tumour pathophysiology[20]. A recent study was performed in 17 patients with PDAC by Joo et al and the sensitivity of FDG PET/MR to assess M stage was $75 \%$ preoperatively[21]. However, there are few studies that used PET/MR radiomics to predict the risk of SDM in pancreatic cancer patients[22]. In addition, features extracted from multiparametric methods including T2WI, DWI, and CE-MRI sequences have lower risk of bias than from one sequence alone[23]. We hypothesize that radiomics signature using features 
derived from ${ }^{18}$ FDG-PET and multiple sequences on MRI may help to predict the risk of SDM in patients with PDAC.

Our study aimed to extract and select the key PET-MRI-based radiomics features which are highly related to the metastasis of PDAC and develop a radiomics signature. And then to construct a radiomics nomogram integrating radiomics signature and clinical factors for predicting the risk of SDM in patients with PDAC, and perform an external test for the model with independent samples obtained from hybrid PET/MR examinations.This nomogram may help clinicians for screening patients at risk of metastasis who require neoadjuvant therapy.

\section{Materials and methods}

\section{Study population}

The joint radiomics model for SDM prediction on PET-MRI was developed on a cohort of 66 patients (development cohort) scanned separately with PET/CT and MRI and validated on an independent cohort of 25 patients (test cohort) imaged with hybrid PET/MR. All of them were pathology-confirmed PDAC patients, which were scanned between 2011 and 2020 at Ruijin Hospital. The patients haven't received any treatment (surgery, chemotherapy, or radiotherapy) before MRI or PET imaging. Here we considered 5 MRI sequences, including contrast-enhanced (CE) T1-weighted imaging (T1WI) on late arterial phase (AP) and portal venous phase (PVP), T2-weighted imaging (T2WI), diffusion-weighted imaging (DWI) and apparent diffusion coefficient (ADC). For the development cohort, ${ }^{18} \mathrm{~F}-\mathrm{FDG}$ PET/CT and MRI examinations were ensured with an interval of less than 30 days.

After the imaging, the SDM was identified by 1) pathology for patients with 
surgery, 2) biopsy for patients with unresectable primary PDAC, 3) the suspicious metastases confirmed by consistent finding at more than two imaging examinations, including contrast-enhanced computed tomography (CECT) of the chest, abdomen and pelvis, MRI of the abdomen, and ${ }^{18} \mathrm{~F}-\mathrm{PET} / \mathrm{CT}$ at the initial diagnosis of PDAC. And size or number of metastases increased or decreased after therapy within 10 months of follow-up. The patients without SDMs were confirmed by no signs of distant metastases through whole-body imaging examinations or no suspicious metastasis during follow-up within 10 months. This retrospective study was approved by our Institutional Review Board, and the requirement to obtain written informed consent was waived. The development cohort was randomly allocated to the training set $(\mathrm{n}=$ $47)$ and testset $(n=19)$ at a ratio of $7: 3$.The clinic-pathological characteristics included age, gender, tumor location, cancer antigen (CA)19-9, CA125, and carcinoembryonic antigen (CEA). The threshold values of CA19-9, CA125, and CEA were $400 \mathrm{U} / \mathrm{mL}[24]$, $35 \mathrm{U} / \mathrm{mL}$, and $5.0 \mathrm{ng} / \mathrm{ml}$, respectively.

\section{${ }^{18}$ F-FDG-PET /CT and MRI protocol}

${ }^{18}$ F-FDG PET/CT was performed on a Discovery STE scanner equipped with a 64-slice CT (GE Healthcare). A 5-6 MBq/kg dose of intravenous injections of ${ }^{18} \mathrm{~F}-\mathrm{FDG}$ was applied after at least 6 hours of fasting. Blood glucose levels were controlled to below $11.0 \mathrm{mmol} / \mathrm{L} .{ }^{18} \mathrm{~F}-\mathrm{PET} / \mathrm{CT}$ scanning was performed at $60 \mathrm{~min} \sim 90 \mathrm{~min}$ after ${ }^{18} \mathrm{~F}$ FDG injections. The scanning image range was from the head to the thigh. Emission images were followed by 2 minutes per bed position for the neck, chest, abdomen and pelvis 5 minutes per bed position for the brain in the three-dimensional acquisition mode. The CT scan data were collected with $148 \mathrm{mAs}$ and $140 \mathrm{kV}$. The section 
thickness of all CT scans was $3.75 \mathrm{~mm}$. CT images and PET images were reconstructed into $512 \times 512$ matrices and $128 \times 128$ matrices, respectively. PET images were reconstructed using ordered-subset expectation-maximization, and the CT images were used for attenuation correction.

Preoperative MR images wereobtained with 1.5 T MRI scanners $(\mathrm{N}=21)$ or 3.0 TMRI scanners $(\mathrm{N}=45)$. The MRI examination included fiveimage sets: axial turbo spin-echo T2 sequence with fat saturation, contrast-enhanced fat-suppressed T1weightedon late arterial phase andportal venous phase,DWI using a single-shot echoplanar imaging pulse sequence with b-values $\left(0,600\right.$ or $\left.800 \mathrm{~s} / \mathrm{mm}^{2}\right)$ and apparent diffusion coefficient (ADC).The ADC was calculated using a monoexponential function with b-values $\left(0 \mathrm{~s} / \mathrm{mm}^{2}\right.$ and 600 or $0 \mathrm{~s} / \mathrm{mm}^{2}$ and $\left.800 \mathrm{~s} / \mathrm{mm}^{2}\right)$. Contrastenhanced imaging was performed with $0.1 \mathrm{mmol} / \mathrm{kg}$ of gadopentetate dimegluminewith intravenous administration at a rate of $2 \mathrm{~mL} / \mathrm{s}$. Late arterial phase and portal venous phase data (35, and 60 seconds post-injection, respectively) were acquired. Supplementary Table 1 reported MRI scan parameters.

\section{PET/MR acquisition}

Whole-body PET/MR was performed using a Siemens Biograph mMR scanner (Siemens Healthcare, Erlangen, Germany). All participants were fasted for atleast 6hours given intravenous ${ }^{18} \mathrm{~F}-\mathrm{FDG} 2.5$ to $6 \mathrm{MBq} / \mathrm{kg} 40-100$ minutes before the study. For whole-body examination, PET was performed from the mid-thighs to the skull base in four bed positions (acquisition time, $4 \mathrm{~min}$ /position) with the patient in a supine armdown position, and the head was scanned with 1 bed position for $8 \mathrm{~min}$. Simultaneous 
MRI with axial T2-weighted 2D half-Fourier acquisition single-shot turbo spin-echo sequences(HASTE), axial DWI with echo-planar sequence(b-values, 50 and 800 $\mathrm{s} / \mathrm{mm} 2$ ), and axial T1-weighted imaging (T1WI) with a DIXON sequence was performed and PET data were acquired at each bed position. The ADC map was calculated using a monoexponential function (b-values, 50 and $800 \mathrm{~s} / \mathrm{mm} 2 ;$ ).

For an abdominal examination, the simultaneous acquisition of PET and MRI data was performed. DCE-MRI was performed with an axialvolumetric interpolated breath-hold examination sequence (TR/TE 4.0/1.2ms, FA $9^{\circ}$, slice thickness $3.1 \mathrm{~mm}$, matrixsize $216 \times 288$, FOV $400 \times 400 \mathrm{~mm}$, NEX 1). Contrast-enhanced dynamic acquisition obtainedafter administration of contrast agent (gadopentetic acid, $0.1 \mathrm{mmol} / \mathrm{kg}$; Magnevist, Bayer) at 20 seconds, 40 seconds, 1 minute, 2 minutesat a flow of $2 \mathrm{~mL} / \mathrm{s}$.

\section{Segmentation and Radiomic features extraction}

Three-dimensional volumes of interest (VOIs) were manually and independently drawn by two nuclear medicine physicians on ${ }^{18} \mathrm{~F}-\mathrm{FDG}-\mathrm{PET} / \mathrm{CT}$ and MRI. Image features were extracted using LIFEx plat form v5.1 for radiomics[25]. All images underwent standardized preprocessing as follows: intensity discretization to 64 gray levels,spatial resampling was set to $2.0 \times 2.0 \times 2.0 \mathrm{~mm}^{3}$.Absolute intensity rescaling using 0-20 SUV units in the ${ }^{18}$ F-FDG-PET. Relative intensity rescalingof MRI was performed using the mean and three standard deviations of the gray levels[26].In total, 212 features including 47 features from PET images and another 205 from MRI images were extracted. For each sequence, the 41 features included 4 shape features, 5 histogram features and 32 texture features. Six conventional metabolic parameters were also 
included in ${ }^{18}$ F-FDG-PET. Details about the extracted features are elaborated in Supplementary Table 2. Intraclass correlation coefficients (ICCs) were calculated to evaluate the interobserver reproducibility of feature extraction.

\section{Construction of the Radiomics Models, Clinical Model and Radiomics Nomogram}

To prevent overfitting in radiomics models, feature subset selection of highdimensional image data was conducted using the following steps. Features with ICCs $>$ 0.75 were retained for subsequent analysis across all sequences to avoid interobserver variability in segmenting the VOIs. The least absolute shrinkage and selection operator (LASSO) logistic regression[27] method was performed to select metastasis-related features of high-dimensional data[28] and to build radiomics model. The penalty parameter tuning was conducted with the smallest 10 -fold cross-validation error. Significant features with nonzero coefficients were selected. A radiomics model was a linear combination of selected features weighted by their respective coefficients. We developed three radiomics models: two were based on features extracted from PET or MRI images, and the third was a combination of both PET and MRI texture parameters (PET-MRI). The features and radiomics models were generated from the training set, and then the testing set was used to confirm our findings in independent cases. The best radiomics signature and clinic-pathological information were used to construct multivariate regression in the training set. The backward stepwise selection was applied by using the likelihood ratio test with Akaike's Information Criterion (AIC) as the stopping rule [29]. Varabilaities with $\mathrm{P}<0.05$ in multivariate logistic regression were introduced to build a radiomics nomogram. The clinical model was based on nomogram 
except for a radiomics signature.

\section{Statistical analyses}

R software (version 3.6.1, http://www.r-project.org)and SPSS 25.0 (IBM, Armonk, NY) were used for performing statistical analysis. $\mathrm{P}<0.05$ (two-sided) was considered statistically significant. Independent sample t-tests and Fisher's exact tests were used for comparing continuous variables and categorical variables between the two groups, respectively. The area under the curve (AUC) of the receiver operator characteristic (ROC) curve was used to evaluate the discrimination performance of the radiomics models in both the training and testing sets. The diagnostic abilities between each two of the three models were compared by using the Delong test[30]. The HosmerLemeshow test was used to assess the goodness-of-fit of the radiomics nomogram. Decision curve analysis (DCA) was used to quantify the net benefit of the models at different threshold probabilities [31].Spearman's correlation coefficient ( $\rho$ ) was calculated to examine the correlation between radiomics features and clinicpathological characteristics. The accuracy, sensitivity and specificity were computed by Confusion matrix-derived metrics. The flow chart of patient recruitment is shown in Fig. 1.

\section{Results}

\section{Patient clinical characteristics}

The clinical characteristics of the 91 patients in the development and test cohorts are summarized in Table 1. Of the patients, 46 had no metastasis and 20 had metastasis in the development cohort, and 14 had no metastasis and 11 had metastasis in the test 
cohort. The ${ }^{18}$ F-FDG PET/CT imaging and MRI showed the sensitivities of $65 \%$ (13 of 20 patients) and $80 \%$ (16 of 20 patients) according to the imaging report in the development cohort, respectively. Two of 20 patients with metastases could not be identified by means of both PET/CT and MRI in the development cohort. Fig.2 showed the case of liver metastasis was not detected in PET/CT and MRI examination in one patient with PDAC. The patient underwent laparotomy eight days after the initial imaging examination. Multiple liver nodules were found during the operation and one nodule was resected and pathologically diagnosed as liver metastasis. Distant metastatic sites were the liver (68\% of patients), peritoneum $(16 \%)$, lung $(10 \%)$, diaphragm (3\%), and supraclavicularlymph node (3\%).

\section{Feature Selection and Radiomics Model Building}

We used the LASSO algorithm to select the optimal subset of radiomics features in the training set. Six radiomics features with nonzero coefficients were selected using PET-MRI images (Fig. 3). Four and Five radiomics features were selected using PET images and MRI images, respectively. The three radiomics models constructed using optimal features weighted respective coefficients by the LASSO regression from each group were as follows:

$$
\begin{aligned}
& \text { Radscore }_{P E T}=0.65 S U V_{\text {peak }}+4.62 \text { Shape_Volume }_{-} 2.12 \text { GLZLM_SZE } \\
& +0.45 \text { GLZLM_LZLGE - } 3.01 \\
& \text { Radscore }_{\text {MRI }}=-0.12 \text { HISTO_Kurtosis }_{T 2}-0.86 \text { Shape_Sphericity }_{T 2} \\
& +0.49 \text { GLZLM_LZHGE } E_{A D C}+2.29 \text { GLRLM_RLNU } U_{A P} \\
& +1.05 \text { GLZLM_SZE } E_{A P}-0.22
\end{aligned}
$$




$$
\begin{aligned}
& \text { Radscore }_{P E T-M R I} \\
& =0.77 S_{\text {Seak }}+3.42 \text { Shape_Volume }_{P E T}+1.54 \text { GLZLM_SZE }_{P E T} \\
& -1.35 \text { Shape_Sphericity }{ }_{T 2}+0.66 \text { GLZLM_LZE } E_{A D C} \\
& -0.24 \text { GLZLM_SZE } E_{A P}-1.68
\end{aligned}
$$

\section{Performance of different radiomics Model}

The ROCs of all three abovementioned radiomics models are provided in Fig. 3Aand 3B. The PET-MRI model yielded the highest discrimination between metastasis and non-metastatis(training: $\mathrm{AUC}=0.94$, test: $\mathrm{AUC}=0.77$ ). The PET model and MRI model yielded an AUC of 0.89 vs. 0.86 in the training group and 0.77 vs. 0.67 in the testgroup. Table 2 shows the diagnostic performance for three radiomics models. The radiomics signature of the PET-MRI with the best performance was being applied to the subsequent analysis.

\section{Development and evaluation of the Radiomic Nomogram}

In the multivariate analysis (Table 3), CA19-9 level, age and the radiomics signature were significantly associated with SDM status. A radiomics nomogram was then constructed with radiomics signature, the CA19-9 level and age (Fig. 5). A clinical model was built using age and CA19-9 levels. The AUC values of the clinical model, radiomics signature, and radiomics nomogram were $0.70,0.89$, and 0.93 , respectively (Fig.6).The accuracy, sensitivity and specificity of radiomics nomogram were $86.3 \%, 87.0 \%$ and $85.0 \%$, respectively. The AUC of radiomics nomogram was statistically significantly higher than the clinical model $(\mathrm{P}<0.001)$ and exhibited similarly to the radiomics signature $(\mathrm{P}>0.05)$ according to the Delong test. The 
calibration curves for the radiomics nomogram (Fig.7) was non-significant $(\mathrm{P}=0.941)$ with the Hosmer-Lemeshow test, showing that good consistency between the estimated and actual metastasis. While the calibration curve of the radiomics signature and clinical model did not show a good fit $(\mathrm{P}<0.05)$. Fig.8 shows the decision curve for three models in predicting synchronous distant metastasis. The radiomics nomogram results bring more benefits than the "treat all" vs. "treat none" scheme. The radiomics nomogram had a higher net benefit than the radiomics signature, clinical model across the majority of the range of threshold probabilities. The radiomics nomogram was evaluated with an independent group (25 patients)to validate the robustness and stability of the model. Its accuracy, sensitivity and specificity were $84.0 \%, 78.6 \%$ and 90.9\%, respectively. The AUC value is 0.85 (95\% confidence interval: $0.69-1.00$ ) (Supplementary Fig 1).

\section{Discussion}

\section{Prediction of SDM from primary tumor}

PDAC is a highly invasive tumor and often leads to distant metastasis. The SDM of pancreatic cancer may be related to the gene changes, tumor microenvironment and other factors. Several altered genes in PDAC, such as, KRAS (90\%), TP53, CDKN2A, and SMAD4[32, 33], were identified to be associated with the invasion and metastasis. Radiomics can unravel the heterogeneity of the tumor [34], which is associated with underlying changes of genetics and tumor microenvironment[35]. The morphological heterogeneity of primary tumors is considered as an important determinant factor of 
metastatic phenotype [36].

The finding of this study confirmed the feasibility of radiomics of primary PDAC tumor to predict the SDM. Radiologically occult metastatic pancreatic cancers were missing by imaging, because distant metastases within 6 months of surgery can be deemed to represent micrometastases that were undetectable during the perioperative period[37]. Thus, establishment of a strategy featuring new imaging modalities to predict metastases missed by preoperative imaging is necessary for patients with a high risk of metastasis. Our findings suggest that PET/MR imaging may be a time-saving and "one-stop-shop" tool to evaluate the SDM in preoperative PDAC patients. And this examination may avoid delaying the time of imaging examination which maybe influence clinical decision making and the outcome of patients. However, there are currently few radiomics studies using PET and multiparametric MRI.

\section{Complementary information between PET and MRI}

PET and MRI decode different (patho-)physiological mechanisms that complement each other and may be accessible through textural feature analysis[38]. A combined, multimodal predictor has the potential to outperform single modality models[39]. This assumption is supported by our findings where the combination of both modalities yielded the highest diagnostic performance compared to the single modality models.

PET and MRI are routine powerful diagnostic tools. The sensitivity of the radiomics signature reached $93.9 \%$ in the training set. Compared with the imaging report, our study found that radiomics signature derived from PET and MRI imaging increase the 
diagnostic sensitivity of SDM in PDAC $\left(65 \%\right.$ for ${ }^{18} \mathrm{~F}-\mathrm{FDG} \mathrm{PET} / \mathrm{CT}$, and $80 \%$ for MRI).In addition, our results from the tumor radiomics setup show that different sequences (i.e., PET, T2, $\mathrm{ADC}$ and $\mathrm{T}_{1} \mathrm{WI}$ in arterial phase) can provide metabolic, anatomic, and functional information. ADC is a quantitative biomarker calculated from diffusion-weighted imaging that can reflect tumor characteristics such as cellular density and aggressiveness[40]. Choi et al demonstrated that entropy was significantly associated with overall survival in patients with PDAC using preoperative $\mathrm{T}_{2} \mathrm{WI}$ texture analysis[41].Previous studies have found integrated PET/MR to be able to assess treatment response[42], prognosis[43] in PDAC patients.

\section{Interpretation of the identified radiomics features}

Radiomics can provide comprehensive tissue and organ characterization undetected by human perception and is a fast, low-cost and noninvasive approach. Our optimal radiomics signature contained 3 features on ${ }^{18} \mathrm{~F}$-FDG PET images and 3 features on MRI. As a conventional metabolic feature,SUVpeak means maximum average SUV in a $1 \mathrm{~cm}^{3}$ sphere of the VOI, which can reflect the metabolic activity. Higher pretreatment SUVpeak in patients with advanced pancreatic cancer indicated a poor prognosis. Kim et al. showed that the SUVpeak was an independent prognostic factor in lung adenocarcinoma[44].The shape-based feature SHAPE_Volume (ml) from PET is the volume of interest. A previous study demonstrated that the probability of tumor dissemination was associated with tumor size at the time of diagnosis[45]. Regarding sphericity on $\mathrm{T}_{2} \mathrm{WI}$, the lower the value, the further the divergence from a sphere. 
Aggressive tumors may have more irregular boundaries and invasion of surrounding tissues on T2WI in patients with non-metastasis than in those with distant metastasis. The remaining texture features, including PET texture features (GLZLM_SZE), ADC texture features (GLZLM_LZE), and AP texture features (GLZLM_SZE) reflect the heterogeneity and image uniformity within the volume of interest.

\section{Value of clinical parameters}

Our study confirmed that the high-performance radiomic nomogram included clinical factors (age and CA19-9 level) and the radiomic signature. Consistent with previous studies, CA-199 was identified as an independent predictor for distant metastasis in a previous study[46]. Dong et al found that the elevated level of CA19-9 was significantly associated with the risk of synchronous liver metastasis in stage IV PDAC patients[47]. Age $>62$ years was correlated with the unresectability of synchronous liver metastasis in patients with PDAC[24]. However, the clinical model included CA19-9 and age achieved an AUC of 0.70 with limited value for the prediction of SDM in PDAC.

\section{$\underline{\text { Robustness of the identified radiomics features }}$}

A critical challenge for the application of radiomics models is robust when applying to different scanners or imaging protocols.Generally, selecting reproducible features across different machines and protocols is important for the development and validation of radiomics models[48]. Often, standardization strategies are recommended to bridge 
the difference of voxel sizes, intensity outlier filtering, intensity discretisation and ICC, which is key to improve reproducibility of radiomic models[49]. In this study, radiomics nomogram was constructed on data from individual PET and MRI and then validated on hybrid PET/MRI scanner. Considering that the images were obtained in different magnet strength $(1.5 \mathrm{~T}, 3.0 \mathrm{~T})$ and with varying parameters, we applied feature normalization to reduce variations among patients.Even though we did not apply harmonization on the radiomics features[50], our current preliminary results demonstrated that certain robustness of the developed methods in cross-scanner applications. Additional data standardization and harmonization may further improve the performance in different scanner and maybe still necessary at extreme situation. Nevertheless, this baseline cross-scanner robustness may be convenient for clinical translation, where the radiomics nomogram may be independently applied in many applications[51].

\section{The application of nomogram}

The predictive nomograms developed in this study may help clinicians quantitatively assess the probability of synchronous distant metastasis and make individualized decisions. For example, a 60-year patient with normal CA19-9 levels and low score radiomics signature is associated less than $10 \%$ probability of having synchronous metastasis, and might benefit from radical surgery. Neoadjuvant therapy are recommended for patients who are at high-risk of distant metastasis but the imaging are not able to reveal the lesion. The benefits of neoadjuvant therapy include eliminating micrometastases, and identifying aggressive tumors to avoid futile surgery 
[52]. At present, whether to carry out neoadjuvant treatment is decided by consulting the surgeon.Our nomogram will identify patients with high-risk metastatic who may benefit from a personalized approach for tumours that are susceptible to neoadjuvant treatment that could extend survival.

\section{$\underline{\text { Limitations }}$}

This study has limitations. First, our data were retrospective, and a small number of patients were involved in this preliminary study due to a few patients receiving imaging examination, larger sample sizes and multicenter evaluation might be needed to validate our radiomics nomogram in the future.Second, The patients included in this study are involved in multiple organ metastases, which can not be used to predict the specific location of distant metastasis in patients with pancreatic ductal adenocarcinoma, but can be used to predict whether metastasis occurs, so as to help patients choose the optimal treatment. Finally, we used only one method for feature selection and classifier. Further investigations may be performed to confirm whether our model be independent of other feature selection and classifier algorithms.

\section{Conclusions}

In summary, our study developed a noninvasive and quantitative tool for the preoperative assessment of the risk of SDM based on multiparametric MRI and PET scans. A radiomics nomogram combining radiomics signature and clinical information may improve the prediction ability of the risk of SDM and provide complementary information for preoperative staging, and optimal therapies in patients with PDAC. And thus, hybridPET/MR imaging with both PET and MRI examinations simultaneously 
might be suggested for "one-stop-shop" preoperative assessing modality in PDAC patients.

\section{Abbreviations}

PDAC: pancreaticadenocarcinoma; SDM: synchronous distant metastasis; ${ }^{18} \mathrm{~F}-\mathrm{FDG}$ PET/CT: ${ }^{18} \mathrm{~F}$-fluorodesoxyglucosepositron emission tomo-graphy/computed tomography; CECT:contrast-enhanced computed tomography; T1WI: T1-weighted imaging;T2WI: T2-weighted imaging; DWI: diffusion-weighted imaging; ADC: apparent diffusion coefficient; AP: arterial phase; PVP: portal venous phase;LASSO: least absolute shrinkage and selection operator; ICC:Intraclass correlation coefficients; ROC: receiver operating characteristic; AUC: area under the curve; CI:confidence interval; DCA:decision curve analysis; VOI: volume of interest; AIC: Akaike information criterion; CEA: serum carcinoembryonic antigen; CA 19-9: serum carbohydrate antigen 19-9.

\section{Contributions}

Study concept and design: XZL, HZ and KYS.Acquisition and segmentation of image: JG, YYB, XYH and XZL. Analysis and interpretation of data: JG and YYB. Clinical studies: FM, BL, M.S, A.R. Draft of the initial manuscript: JG and YYB. Further review and editing of the manuscript: XZL, HZ, W.W, M.S and KYS. All authors read and approved the final manuscript.

\section{Funding information}

This study was supported by research grants from the National Natural Science Foundation of China (62073218), Shanghai Science and Technology Committee of 
Shanghai Municipality(20Y11912300) and Shanghai Municipal Key Clinical Specialty

(shslczdzk03403).

\section{Compliance with ethical standards}

Conflict of interest All other authors have no conflicts of interest.

Research involving human participants All procedures performed in studies involving human participants were in accordance with the ethical standards of the institutional and/or national research committee and with the 1964 Helsinki declaration and its later amendments or comparable ethical standards.

Informed consent Informed consent was waived for this retrospective study.

\section{References:}

1. Rawla P, Sunkara T, Gaduputi V. Epidemiology of Pancreatic Cancer: Global Trends, Etiology and Risk Factors. World J Oncol. 2019;10:10-27. doi:10. 14740/wjon1166.

2. Siegel RL, Miller KD, Jemal A. Cancer statistics, 2019. CA Cancer J Clin. 2019;69:7-34. doi:10. 3322/caac. 21551.

3. Louvet C, Philip PA. Accomplishments in 2007 in the treatment of metastatic pancreatic cancer. Gastrointest Cancer Res. 2008;2:S37-41.

4. Shi S, Yu XJ. Time to think: Selecting patients who may benefit from synchronous resection of primary pancreatic cancer and liver metastases. World journal of gastroenterology. 2018;24:3677-80. doi:10.3748/wjg. v24. i33. 3677.

5. Ge J, Li L, Ma Z, Jiang B, Yuan C, Wang H, et al. A nomogram of preoperative predictors for occult metastasis in patients with PDAC during laparoscopic exploration. Gland surgery. 2021;10:279-89. doi:10.21037/gs-20-605.

6. Campbel1 PJ, Yachida S, Mudie LJ, Stephens PJ, Pleasance ED, Stebbings LA, et al. The patterns and dynamics of genomic instability in metastatic pancreatic cancer. Nature. 2010;467:1109-13. doi:10. 1038/nature09460.

7. NCC. N. Pancreatic Adenocarcinoma(Version 2.2021). 2021; Available online at: https://www. nccn. org/professionals/physician_gls/pdf/pancreatic.pdf.

8. Kauhanen SP, Komar G, Seppänen MP, Dean KI, Minn HR, Kajander SA, et al. A prospective diagnostic accuracy study of $18 \mathrm{~F}$-fluorodeoxyglucose positron emission tomography/computed tomography, multidetector row computed tomography, and magnetic resonance imaging in primary diagnosis and staging of pancreatic cancer. Annals of surgery. 2009;250:957-63. doi:10. 1097/SLA. 0b013e3181b2fafa.

9. Asagi A, Ohta K, Nasu J, Tanada M, Nadano S, Nishimura R, et al. Utility of contrast-enhanced FDG-PET/CT in the clinical management of pancreatic cancer: impact 
on diagnosis, staging, evaluation of treatment response, and detection of recurrence. Pancreas. 2013;42:11-9. doi:10. 1097/MPA. 0b013e3182550d77.

10. Wang XY, Yang F, Jin C, Fu DL. Utility of PET/CT in diagnosis, staging, assessment of resectability and metabolic response of pancreatic cancer. World journal of gastroenterology. 2014;20:15580-9. doi:10. 3748/wjg. v20. i42. 15580.

11. Sahani DV, Bonaffini PA, Catalano OA, Guimaraes AR, Blake MA. State-of-the-art PET/CT of the pancreas: current role and emerging indications. Radiographics : a review publication of the Radiological Society of North America, Inc. 2012;32:113358; discussion 58-60. doi:10. 1148/rg. 324115143.

12. Motosugi U, Ichikawa T, Morisaka H, Sou H, Muhi A, Kimura K, et al. Detection of pancreatic carcinoma and liver metastases with gadoxetic acid-enhanced MR imaging: comparison with contrast-enhanced multi-detector row CT. Radiology. 2011;260:446-53. doi:10. 1148/radiol. 11103548.

13. Holzapfel K, Reiser-Erkan C, Fingerle AA, Erkan M, Eiber MJ, Rummeny EJ, et al. Comparison of diffusion-weighted MR imaging and multidetector-row CT in the detection of liver metastases in patients operated for pancreatic cancer. Abdom Imaging. 2011;36:179-84. doi:10. 1007/s00261-010-9633-5.

14. Miller FH, Rini NJ, Keppke AL. MRI of adenocarcinoma of the pancreas. AJR Am J Roentgenol. 2006;187:W365-74. doi:10. 2214/ajr. 05. 0875.

15. Diederichs CG, Staib L, Vogel J, Glasbrenner B, Glatting G, Brambs HJ, et al. Values and limitations of $18 \mathrm{~F}$-fluorodeoxyglucose-positron-emission tomography with preoperative evaluation of patients with pancreatic masses. Pancreas. 2000;20:10916. doi:10. 1097/00006676-200003000-00001.

16. Karaosmanoglu AD, Onur MR, Ozmen MN, Akata D, Karcaaltincaba M. Magnetic Resonance Imaging of Liver Metastasis. Seminars in ultrasound, CT, and MR. 2016;37:533-48. doi:10. 1053/j. sult. 2016. 08. 005.

17. Lambin P, Leijenaar RTH, Deist TM, Peerlings J, de Jong EEC, van Timmeren J, et al. Radiomics: the bridge between medical imaging and personalized medicine. Nature reviews Clinical oncology. 2017;14:749-62. doi:10. 1038/nrclinonc. 2017. 141.

18. Rios Velazquez E, Parmar C, Liu Y, Coroller TP, Cruz G, Stringfield 0, et al. Somatic Mutations Drive Distinct Imaging Phenotypes in Lung Cancer. Cancer Res. 2017; $77: 3922-30$. doi:10. 1158/0008-5472. Can-17-0122.

19. He C, Zhong L, Zhang Y, Cai Z, Lin X. Development and validation of a nomogram to predict liver metastasis in patients with pancreatic ductal adenocarcinoma: a large cohort study. Cancer management and research. 2019;11:3981-91. doi: 10. 2147/cmar. S200684.

20. O’Connor JP, Jackson A, Asselin MC, Buckley DL, Parker GJ, Jayson GC. Quantitative imaging biomarkers in the clinical development of targeted therapeutics: current and future perspectives. The Lancet Oncology. 2008;9:766-76. doi:10. 1016/s1470-2045(08) 70196-7.

21. Joo I, Lee JM, Lee DH, Lee ES, Paeng JC, Lee SJ, et al. Preoperative Assessment of Pancreatic Cancer with FDG PET/MR Imaging versus FDG PET/CT Plus Contrast-enhanced Multidetector CT: A Prospective Preliminary Study. Radiology. 2017;282:149-59. doi:10. 1148/radiol. 2016152798. 
22. Gao J, Huang X, Meng H, Zhang M, Zhang X, Lin X, et al. Performance of Multiparametric Functional Imaging and Texture Analysis in Predicting Synchronous Metastatic Disease in Pancreatic Ductal Adenocarcinoma Patients by Hybrid PET/MR: Initial Experience. Frontiers in oncology. 2020;10:198. doi:10. 3389/fonc. 2020. 00198. 23. Zhang B, Tian J, Dong D, Gu D, Dong Y, Zhang L, et al. Radiomics Features of Multiparametric MRI as Novel Prognostic Factors in Advanced Nasopharyngeal Carcinoma. Clin Cancer Res. 2017;23:4259-69. doi:10. 1158/1078-0432. Ccr-16-2910.

24. Shi HJ, Jin C, Fu DL. Preoperative evaluation of pancreatic ductal adenocarcinoma with synchronous liver metastasis: Diagnosis and assessment of unresectability. World journal of gastroenterology. 2016;22:10024-37. doi:10. 3748/wjg. v22. i45. 10024.

25. Nioche C, Orlhac F, Boughdad S, Reuzé S, Goya-0uti J, Robert C, et al. LIFEx: A Freeware for Radiomic Feature Calculation in Multimodality Imaging to Accelerate Advances in the Characterization of Tumor Heterogeneity. Cancer Res. 2018;78:47869. doi:10. 1158/0008-5472. Can-18-0125.

26. Collewet G, Strzelecki M, Mariette F. Influence of MRI acquisition protocols and image intensity normalization methods on texture classification. Magn Reson Imaging. 2004;22:81-91. doi:10. 1016/j. mri. 2003. 09. 001.

27. Tibshirani R. Regression shrinkage and selection via the lasso: a retrospective. 2011;73:273-82. doi:10. 1111/j. 1467-9868. 2011. 00771. x.

28. Sauerbrei W, Royston P, Binder H. Selection of important variables and determination of functional form for continuous predictors in multivariable model building. Stat Med. 2007;26:5512-28. doi:10.1002/sim. 3148.

29. Sauerbrei W, Boulesteix AL, Binder H. Stability investigations of multivariable regression models derived from low- and high-dimensional data. Journal of biopharmaceutical statistics. 2011;21:1206-31. doi:10. 1080/10543406.2011.629890.

30. DeLong ER, DeLong DM, Clarke-Pearson DL. Comparing the areas under two or more correlated receiver operating characteristic curves: a nonparametric approach. Biometrics. 1988;44:837-45.

31. Vickers AJ, Elkin EB. Decision curve analysis: a novel method for evaluating prediction models. Medical decision making : an international journal of the Society for Medical Decision Making. 2006;26:565-74. doi:10. 1177/0272989x06295361.

32. Hahn WC, Counter CM, Lundberg AS, Beijersbergen RL, Brooks MW, Weinberg RA. Creation of human tumour cells with defined genetic elements. Nature. 1999;400:4648. doi:10. 1038/22780.

33. Roberts NJ, Norris AL, Petersen GM, Bondy ML, Brand R, Gallinger S, et al. Whole Genome Sequencing Defines the Genetic Heterogeneity of Familial Pancreatic Cancer. Cancer discovery. 2016;6:166-75. doi:10. 1158/2159-8290. Cd-15-0402.

34. Hou Z, Yang Y, Li S, Yan J, Ren W, Liu J, et al. Radiomic analysis using contrastenhanced CT: predict treatment response to pulsed low dose rate radiotherapy in gastric carcinoma with abdominal cavity metastasis. Quantitative imaging in medicine and surgery. 2018;8:410-20. doi:10.21037/qims. 2018. 05. 01.

35. Yoon HJ, Kang J, Park H, Sohn I, Lee SH, Lee HY. Deciphering the tumor microenvironment through radiomics in non-small cell lung cancer: Correlation with immune profiles. PloS one. 2020;15:e0231227. doi:10. 1371/journal. pone. 0231227. 
36. Zhao ZM, Zhao B, Bai Y, Iamarino A, Gaffney SG, Schlessinger J, et al. Early and multiple origins of metastatic lineages within primary tumors. Proceedings of the National Academy of Sciences of the United States of America. 2016;113:2140-5. doi: 10. 1073/pnas. 1525677113.

37. Oba A, Inoue Y, Ono Y, Irie S, Sato T, Mise Y, et al. Radiologically occult metastatic pancreatic cancer: how can we avoid unbeneficial resection? Langenbeck's archives of surgery. 2020;405:35-41. doi:10. 1007/s00423-019-01846-2.

38. Feng Q, Liang J, Wang L, Niu J, Ge X, Pang P, et al. Radiomics Analysis and Correlation With Metabolic Parameters in Nasopharyngeal Carcinoma Based on PET/MR Imaging. Frontiers in oncology. 2020;10:1619. doi:10.3389/fonc. 2020. 01619.

39. Cheng SH, Liu D, Hou B, Hu Y, Huo L, Xing H, et al. PET-MR Imaging and MR Texture Analysis in the Diagnosis of Pancreatic Cysts: A Prospective Preliminary Study. Academic radiology. 2020;27:996-1005. doi:10. 1016/j. acra. 2019. 09. 001.

40. Charles-Edwards EM, deSouza NM. Diffusion-weighted magnetic resonance imaging and its application to cancer. Cancer Imaging. 2006;6:135-43. doi:10.1102/14707330. 2006. 0021.

41. Choi MH, Lee YJ, Yoon SB, Choi JI, Jung SE, Rha SE. MRI of pancreatic ductal adenocarcinoma: texture analysis of T2-weighted images for predicting long-term outcome. Abdom Radiol (NY). 2019;44:122-30. doi:10. 1007/s00261-018-1681-2.

42. Wang ZJ, Behr S, Consunji MV, Yeh BM, Ohliger MA, Gao K, et al. Early Response Assessment in Pancreatic Ductal Adenocarcinoma Through Integrated PET/MRI. AJR American journal of roentgenology. 2018;211:1010-9. doi:10.2214/ajr. 18. 19602.

43. Chen BB, Tien YW, Chang MC, Cheng MF, Chang YT, Yang SH, et al. Multiparametric PET/MR imaging biomarkers are associated with overall survival in patients with pancreatic cancer. European journal of nuclear medicine and molecular imaging. 2018;45:1205-17. doi:10. 1007/s00259-018-3960-0.

44. Kim YI, Paeng JC, Park YS, Cheon GJ, Lee DS, Chung JK, et al. Relation of EGFR Mutation Status to Metabolic Activity in Localized Lung Adenocarcinoma and Its Influence on the Use of FDG PET/CT Parameters in Prognosis. AJR Am J Roentgenol. 2018;210:1346-51. doi:10. 2214/ajr. 17. 18916.

45. Ansari D, Bauden M, Bergström S, Rylance R, Marko-Varga G, Andersson R. Relationship between tumour size and outcome in pancreatic ductal adenocarcinoma. $\mathrm{Br}$ J Surg. 2017;104:600-7. doi:10. 1002/bjs. 10471.

46. van Manen L, Groen JV, Putter H, Vahrmeijer AL, Swijnenburg RJ, Bonsing BA, et al. Elevated CEA and CA19-9 serum levels independently predict advanced pancreatic cancer at diagnosis. Biomarkers : biochemical indicators of exposure, response, and susceptibility to chemicals. 2020;25:186-93. doi:10.1080/1354750x. 2020. 1725786.

47. Santhosh S, Mittal BR, Bhasin DK, Rana SS, Gupta R, Das A, et al. Fluorodeoxyglucose-positron emission tomography/computed tomography performs better than contrast-enhanced computed tomography for metastasis evaluation in the initial staging of pancreatic adenocarcinoma. Annals of nuclear medicine. 2017;31:575-81. doi:10. 1007/s12149-017-1193-0.

48. Hatt M, Tixier F, Pierce L, Kinahan PE, Le Rest CC, Visvikis D. Characterization of PET/CT images using texture analysis: the past, the present $\cdots$ any future? European 
journal of nuclear medicine and molecular imaging. 2017;44:151-65. doi:10. 1007/s00259-016-3427-0.

49. Zwanenburg A, Vallières M, Abdalah MA, Aerts H, Andrearczyk V, Apte A, et al. The Image Biomarker Standardization Initiative: Standardized Quantitative Radiomics for High-Throughput Image-based Phenotyping. Radiology. 2020;295:328-38. doi:10. 1148/radiol. 2020191145.

50. Da-Ano R, Visvikis D, Hatt M. Harmonization strategies for multicenter radiomics investigations. Physics in medicine and biology. 2020. doi:10. 1088/1361-6560/aba798. 51. Lucia F, Visvikis D, Vallières M, Desseroit MC, Miranda 0, Robin P, et al. External validation of a combined PET and MRI radiomics model for prediction of recurrence in cervical cancer patients treated with chemoradiotherapy. European journal of nuclear medicine and molecular imaging. 2019;46:864-77. doi:10. 1007/s00259-018-4231-9.

52. Heinrich S, Lang H. Neoadjuvant Therapy of Pancreatic Cancer: Definitions and Benefits. International journal of molecular sciences. 2017;18. doi:10. 3390/i jms18081622. 
Table 1 Clinical and pathological analyses of pancreatic ductal adenocarcinoma patients with or without metastasis

\begin{tabular}{|c|c|c|c|c|c|c|}
\hline \multirow[b]{2}{*}{ Characteristic } & \multicolumn{3}{|c|}{ Development cohort $(n=66)$} & \multicolumn{3}{|c|}{ Test $\operatorname{cohort}(n=25)$} \\
\hline & $\begin{array}{l}\text { Non- } \\
\text { metastasis } \\
(\mathrm{n}=46)\end{array}$ & $\begin{array}{l}\text { metastasis } \\
(\mathrm{n}=20)\end{array}$ & $\begin{array}{l}\mathrm{P} \\
\text { value }\end{array}$ & $\begin{array}{l}\text { Non- } \\
\text { metastasis } \\
(\mathrm{n}=14)\end{array}$ & $\begin{array}{l}\text { metastasis } \\
(\mathrm{n}=11)\end{array}$ & $\begin{array}{l}\mathrm{P} \\
\text { value }\end{array}$ \\
\hline $\operatorname{Age}(y)$ & & & 0.593 & & & 1.0 \\
\hline$<60$ & 23 & 8 & & 5 & 4 & \\
\hline$\geq 60$ & 23 & 12 & & 9 & 7 & \\
\hline Sex & & & 0.189 & & & 0.115 \\
\hline Female & 17 & 11 & & 3 & 6 & \\
\hline Male & 29 & 9 & & 11 & 5 & \\
\hline \multicolumn{7}{|l|}{ Clinical tumor stage } \\
\hline I- II & 28 & 0 & & 13 & 0 & \\
\hline III- IV & 18 & 20 & & 1 & 11 & \\
\hline $\begin{array}{l}\text { Location of primary } \\
\text { tumor }\end{array}$ & & & 0.032 & & & 0.116 \\
\hline Head/neck & 32 & 6 & & 10 & 4 & \\
\hline Body/tail & 14 & 14 & & 4 & 7 & \\
\hline \multicolumn{7}{|l|}{$\begin{array}{l}\text { Confirmation } \\
\text { approaches of } \\
\text { metastases }\end{array}$} \\
\hline By pathology & - & 13 & & & 7 & \\
\hline By follow-up & - & 7 & & & 4 & \\
\hline \multicolumn{7}{|l|}{ Metastatic site } \\
\hline Liver & - & 14 & & & 7 & \\
\hline Peritoneum & - & 3 & & & 2 & \\
\hline Lung & - & 2 & & & 1 & \\
\hline Diaphragm & - & 1 & & & 0 & \\
\hline $\begin{array}{l}\text { Supraclavicular lymph } \\
\text { node }\end{array}$ & - & 0 & & & 1 & \\
\hline CEA, $\mathrm{ng} / \mathrm{ml}$ & & & 0.174 & & & 0.661 \\
\hline$<5$ & 30 & 9 & & 10 & 9 & \\
\hline$\geq 5$ & 16 & 11 & & 4 & 2 & \\
\hline CA125, U/mL & & & 0.008 & & & 0.177 \\
\hline$<35$ & 37 & 9 & & 12 & 6 & \\
\hline$\geq 35$ & 9 & 11 & & 2 & 5 & \\
\hline CA199, U/mL & & & 0.014 & & & 0.017 \\
\hline$<400$ & 32 & 7 & & 12 & 4 & \\
\hline$\geq 400$ & 14 & 13 & & 2 & 7 & \\
\hline Diabetes mellitus & & & 0.118 & & & 0.208 \\
\hline No & 32 & 18 & & 7 & 2 & \\
\hline Yes & 14 & 2 & & 7 & 9 & \\
\hline
\end{tabular}

$\mathrm{CEA}=$ carcinoembryonic antigen; CA 19-9 carbohydrate antigen 19-9 
Table2 Pridiction performance of three radiomics model for synchromous distant metastasis in preoperative pancreatic ductal adenocarcinoma

\begin{tabular}{|c|c|c|c|c|c|c|}
\hline \multirow{2}{*}{$\begin{array}{l}\text { Models } \\
\text { Set }\end{array}$} & \multicolumn{2}{|c|}{ PET model } & \multicolumn{2}{|c|}{ MRI model } & \multicolumn{2}{|c|}{$\overline{\text { PET-MR model }}$} \\
\hline & training & test & training & test & training & test \\
\hline Accuracy & 0.894 & 0.789 & 0.830 & 0.737 & 0.914 & 0.737 \\
\hline Sensitivity & 1.000 & 0.692 & 0.879 & 0.846 & 0.939 & 0.615 \\
\hline Specificity & 0.643 & 1.000 & 0.714 & 0.500 & 0.857 & 1.000 \\
\hline Feature number & 4 & & 5 & & 6 & \\
\hline
\end{tabular}


Table3. Useful features for predicting synchromous distant metastasis of preoperative PDAC in the nomoram

\begin{tabular}{llllll}
\hline Variable & \multicolumn{3}{l}{ Multivariate logistic regression } & \multicolumn{2}{l}{ Nomogram } \\
\cline { 2 - 6 } & OR (95\% CI) & P value & $\beta$ & OR (95\% CI) & P value \\
\hline $\begin{array}{l}\text { Age }(<60 \\
\text { vs. } \geq 60)\end{array}$ & $2.71(0.55-5.78)$ & 0.034 & 4.73 & $1.55(-0.16-3.79)$ & 0.103 \\
$\begin{array}{l}\text { CA125, } \\
\text { U/mL }\end{array}$ & $1.89(-0.20-4.47)$ & 0.098 & & & \\
CA 19-9, & $2.09(0.16-4.49)$ & 0.049 & 5.34 & $1.68(0.12-3.49)$ & 0.045 \\
U/mL & $-2.31(-5.60-0.11)$ & 0.094 & & & \\
$\begin{array}{l}\text { Diabetes } \\
\text { mellitus }\end{array}$ & & & & \\
$\begin{array}{l}\text { Radiomics } \\
\text { signature }\end{array}$ & $3.66(1.89-6.44)$ & 0.001 & 30.03 & $3.40(1.87-5.70)$ & $<0.001$ \\
\hline $\begin{array}{l}\text { OR odds ratio; CI confidence interval; CEA=carcinoembryonic antigen; CA 19-9 carbohydrate } \\
\text { antigen 19-9 }\end{array}$ & & & &
\end{tabular}




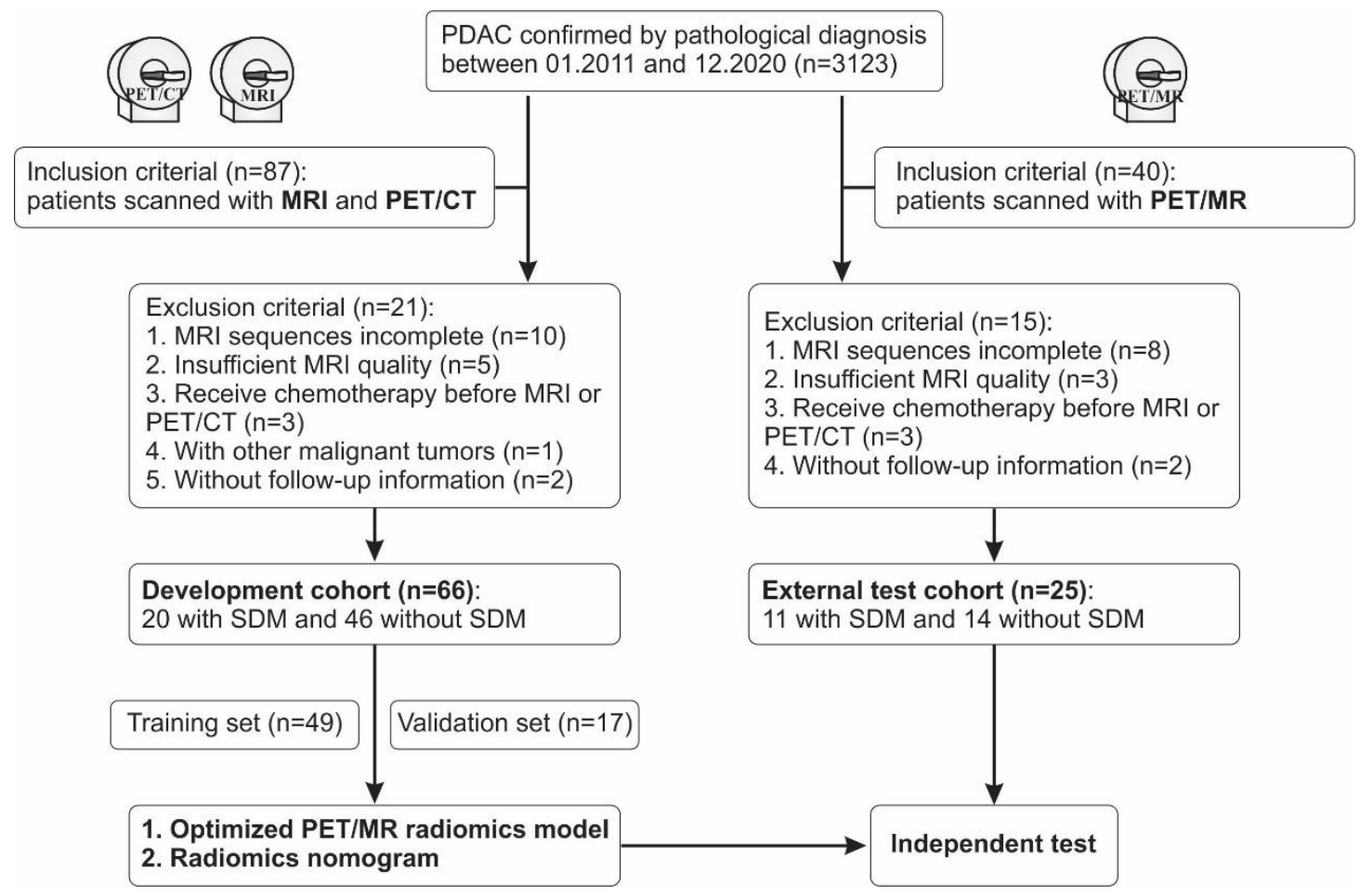

Fig.1 The flow chart of patient recruitment predicting the SDM of patients with PDAC using PET and MRI images. SDM: Synchronous distant metastasis; PDAC: pancreatic ductal adenocarcinoma 


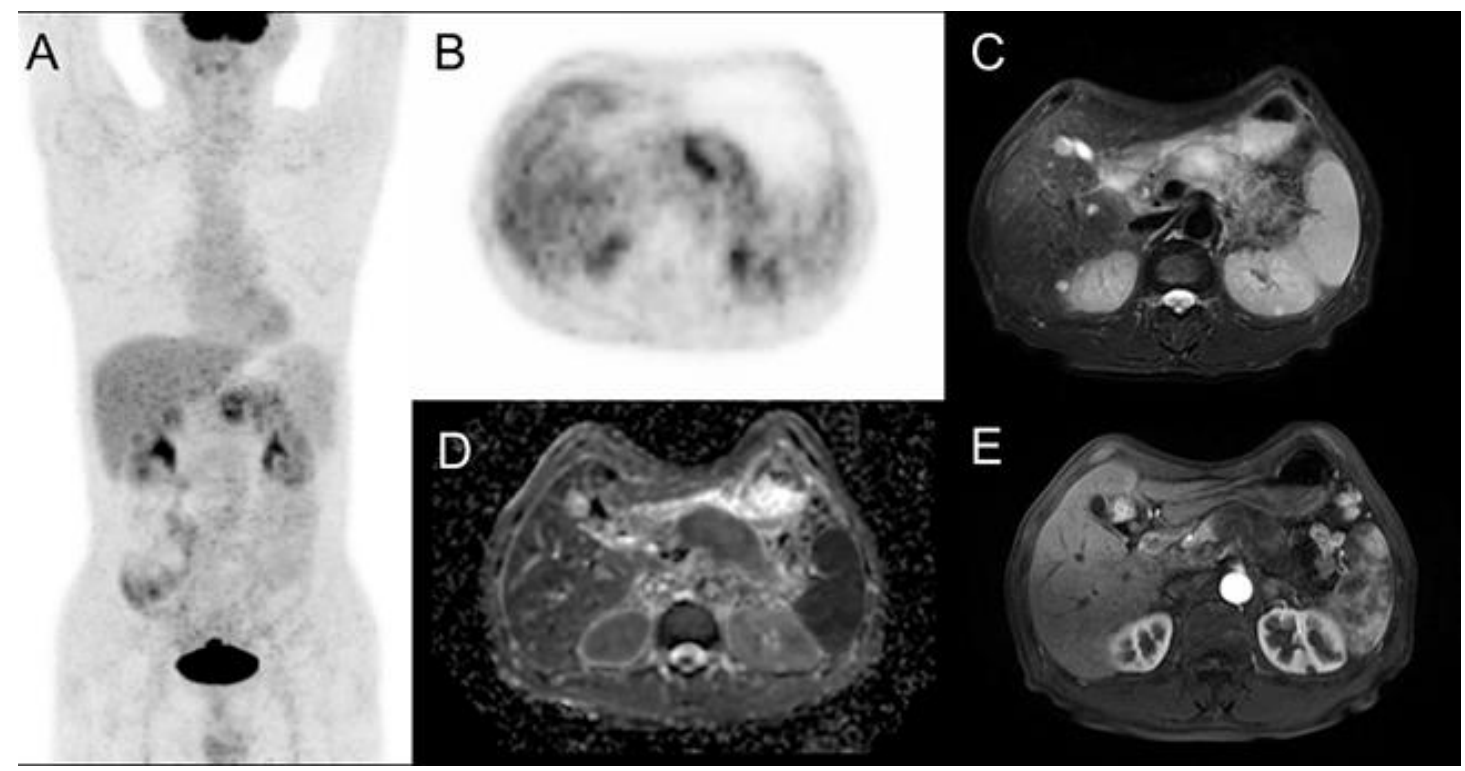

Fig.2An example illustrating the complementary value of radiomics nomogram in predicting synchronous distant metastasis in a 67-year-old male patient with metastatic pancreatic adenocarcinoma (false-negative on PET/CT and MRI).(A) Whole-body maximum intensity projection (MIP) images and (B) axial abdominal PET image showed elevated tracer uptake in the body of the pancreas. Axial T2WI with fat saturation (C) showed hyperintense lesion and an ill-defined hypointense on (D) apparent diffusion coefficient (ADC) map and (E) contrast-enhanced T1weighted image with fat suppression (fs) on late arterial phase is seen in the body of the pancreas.There were no suspicious hepatic metastasis. PET images (A) and (B) showed no evidence of metastatic foci, hyper-intensity on T2WI (C), no hypointense lesion on ADC map (D), and low signals on T1WI+fs (E) in the liver parenchyma.Based on these findings, the liver lesions were rated as multiple hepatic cysts.Liver metastasis was pathologically confirmed via laparotomy. The results of the laboratory examination and radiomics indicated that (CA19-9) was $306.4 \mathrm{U} / \mathrm{mL}$, SUVpeak=5.18,PETSHAPE_volume=39.82,PETGLZLM_SZE=0.38,T2SHAPE_Sph ericity=0.92, ADCGLZLM_LZE=90.3,APGLZLM_SZE=0.73.And this patient was predicted as SDM by radiomics nomogram with high risk (0.99). 
A

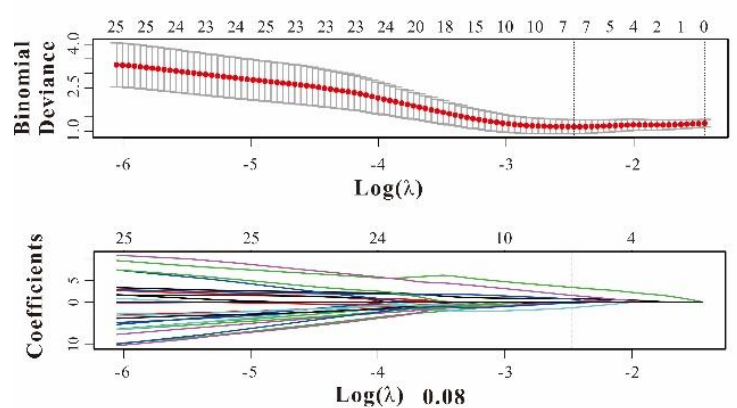

C

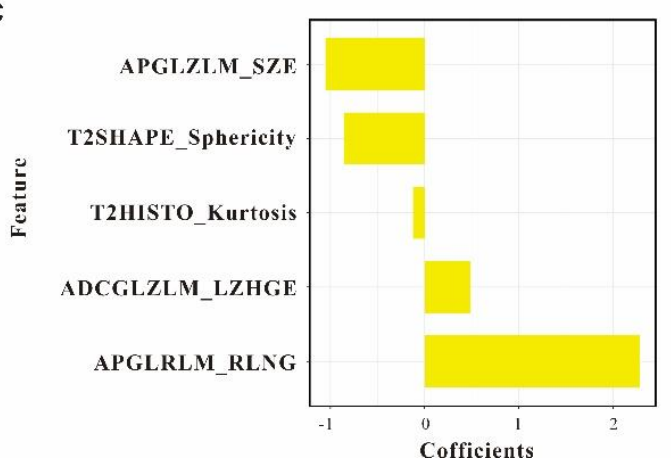

B

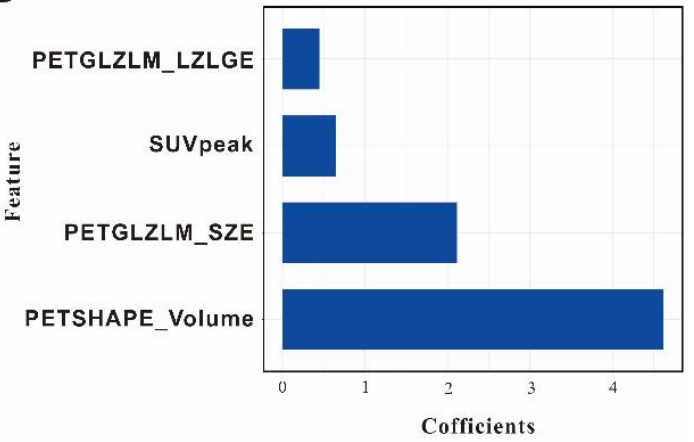

D

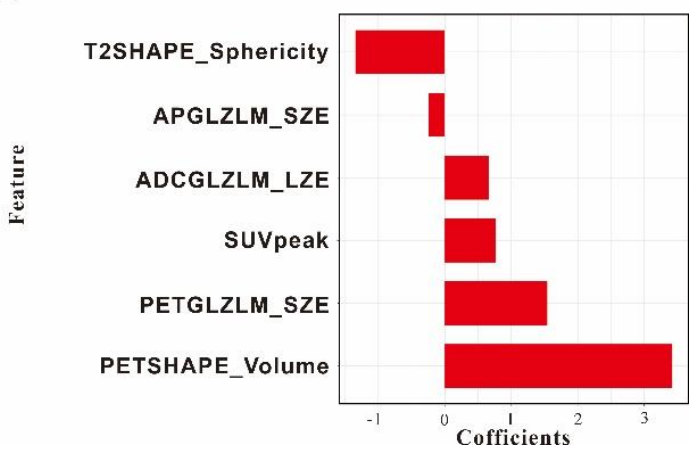

Fig.3The optimal subset of radiomics feature selection using least absolute shrinkage and selection operator (LASSO) algorithm based on the PET and MR images. The 10fold cross-validation were used to extract the optimal subset of radiomic features. A.Tuning parameter $(\lambda)$ selection in LASSO involved the use of 10 -fold crossvalidation with minimum criteria. The optimal $\lambda$ value of 0.0846 was selected. 6 features with nonzero coefficients using the optimal $\lambda$ are shown in the plot. B.The roles of 4 features with nonzero coefficients that contributed to the PET model are represented. C. MRI model was established with 5 features. D. 6 selected features of PET-MRI model was shown. 

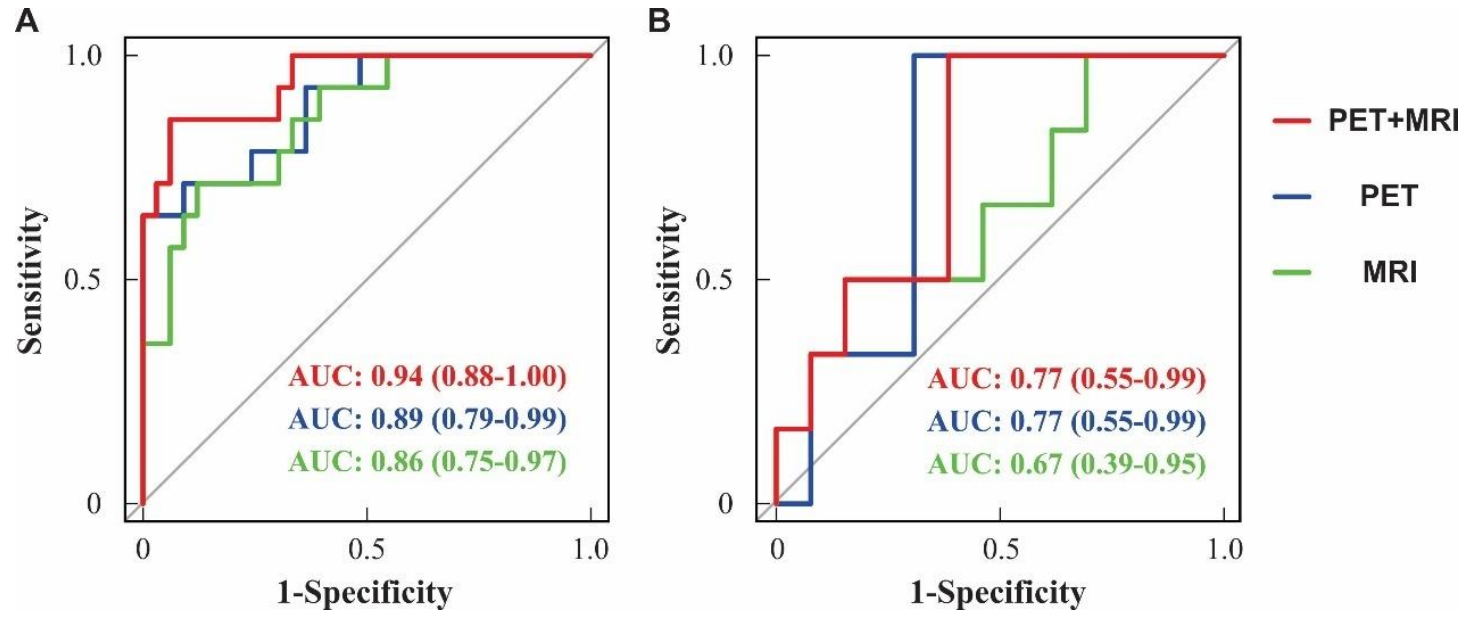

Fig.4 The receiver operator characteristic (ROC) curves for the PET model, the MRI model, and the joint PET-MRI model in differentiating distant metastasis status. A.The ROC curve of the training set. B. The ROC curve of the test set. 

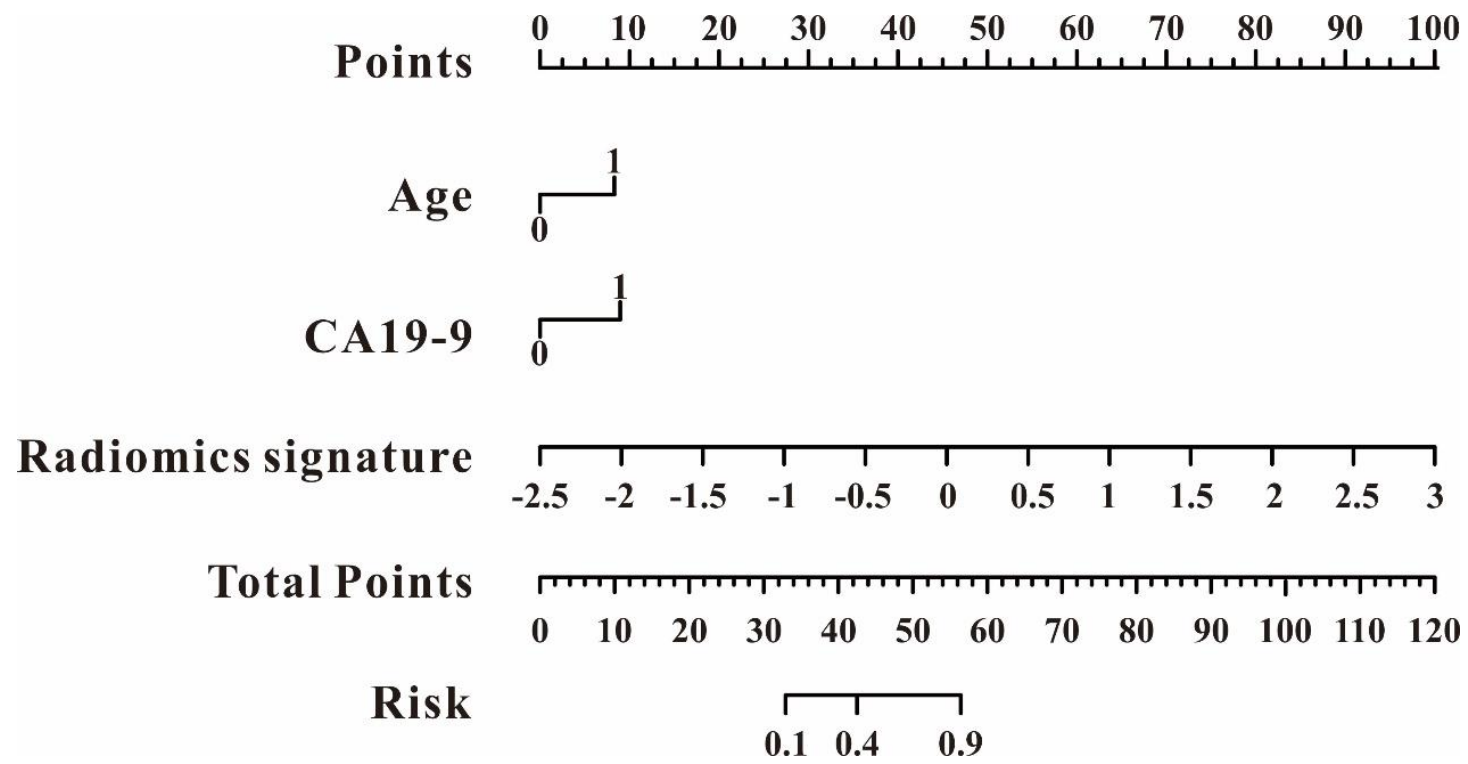

Fig. 5The radiomic nomogram incorporating the age, CA19-9 level and radiomics signature for predicting synchronous distant metastasis in pancreatic ductal adenocarcinoma patients. 


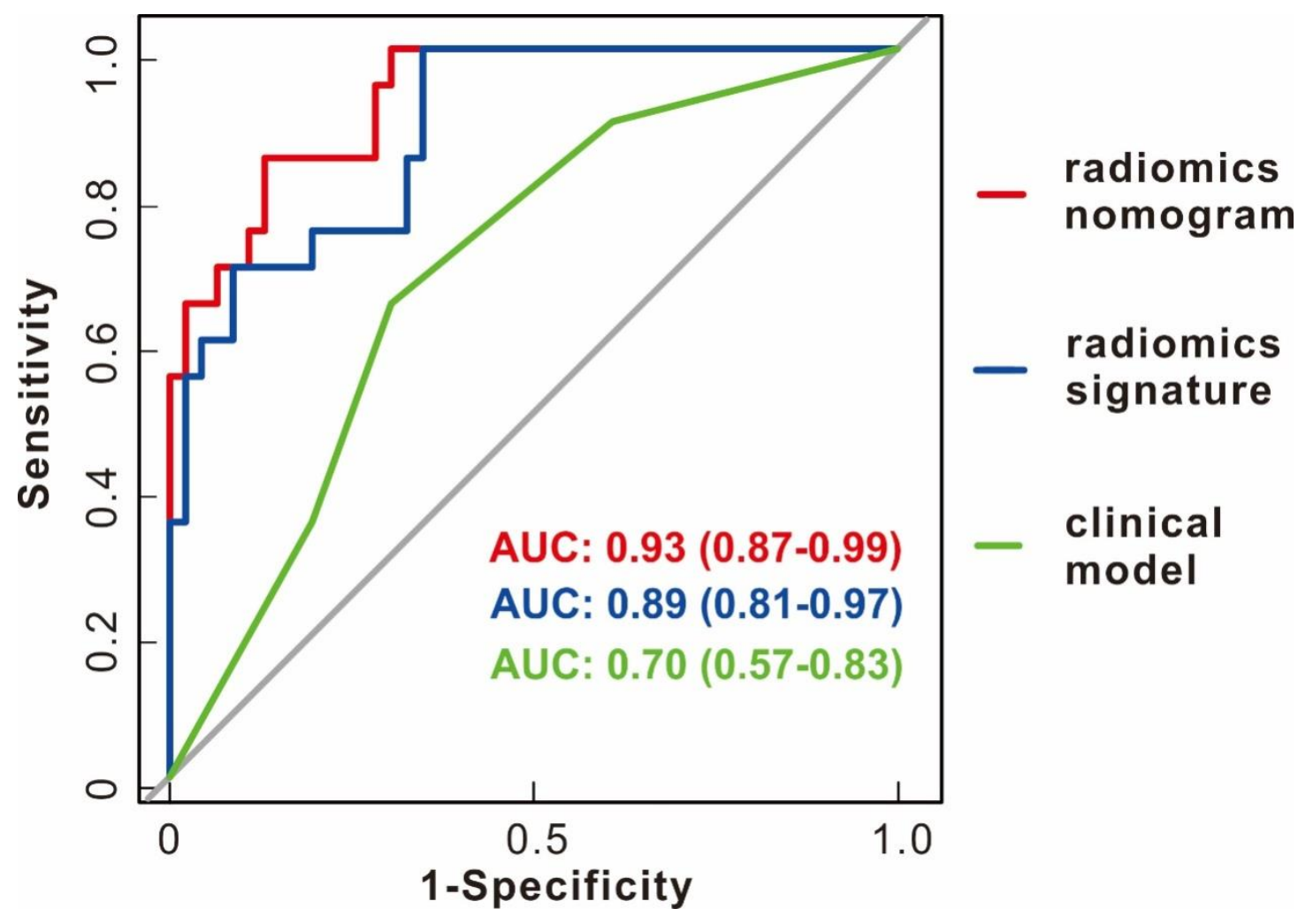

Fig.6 The receiver operator characteristic curves of the three predictive models in diagnosing synchronous distant metastasis in entire pancreatic adenocarcinoma patients. AUC: area under the curve 


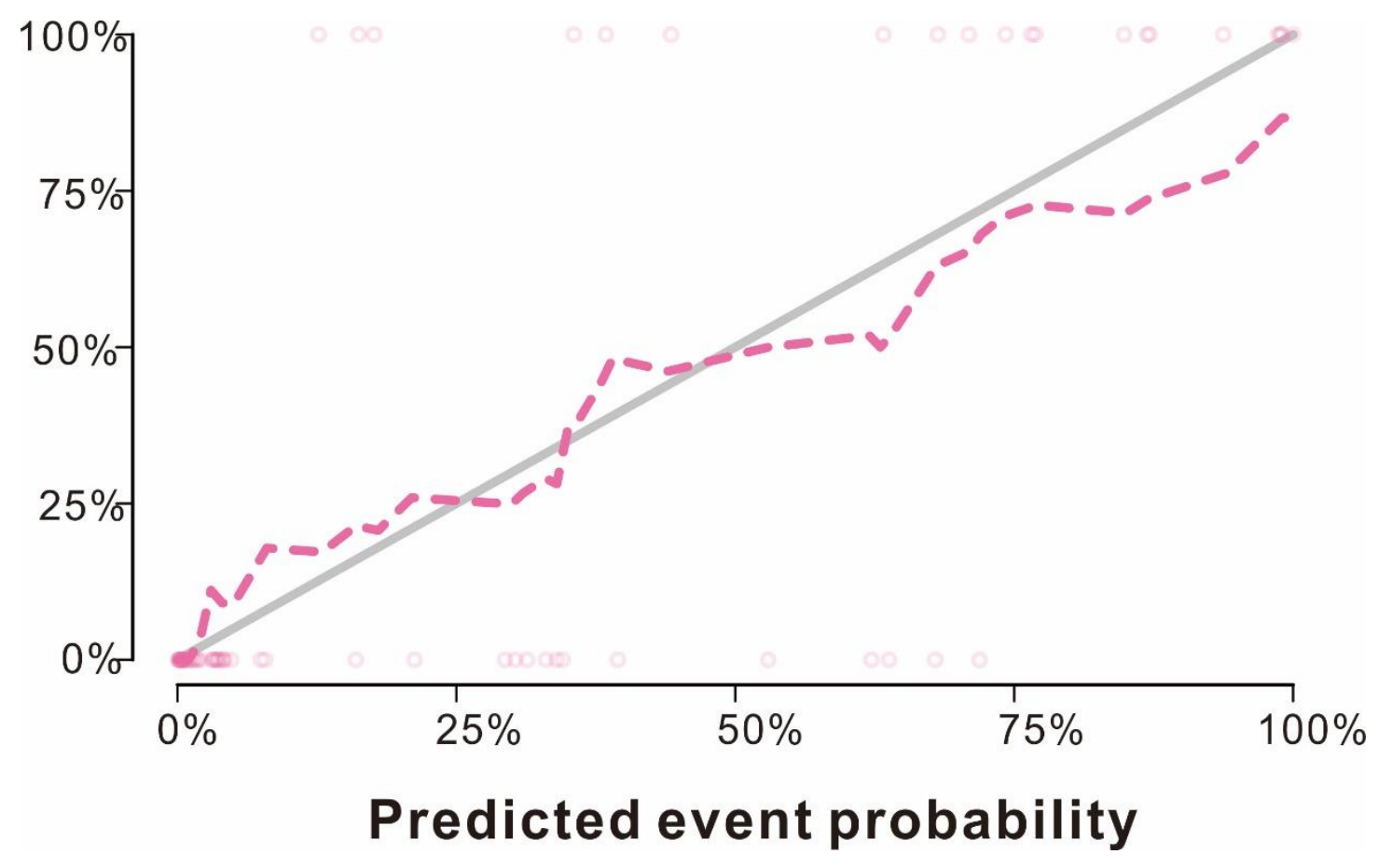

Fig.7 Calibration curves of the radiomics nomogram. The y-axis represents the actual rate of the metastasis and the $\mathrm{x}$-axis reprents the estimated rate of metastasis. The black line on the diagonal indicates that the perfect agreement between the predicted value and the actual value. The dotted lines represent the predictive performance of radiomics nomogram. The closer the dotted line appear along the diagonal line, the better calibrated. 


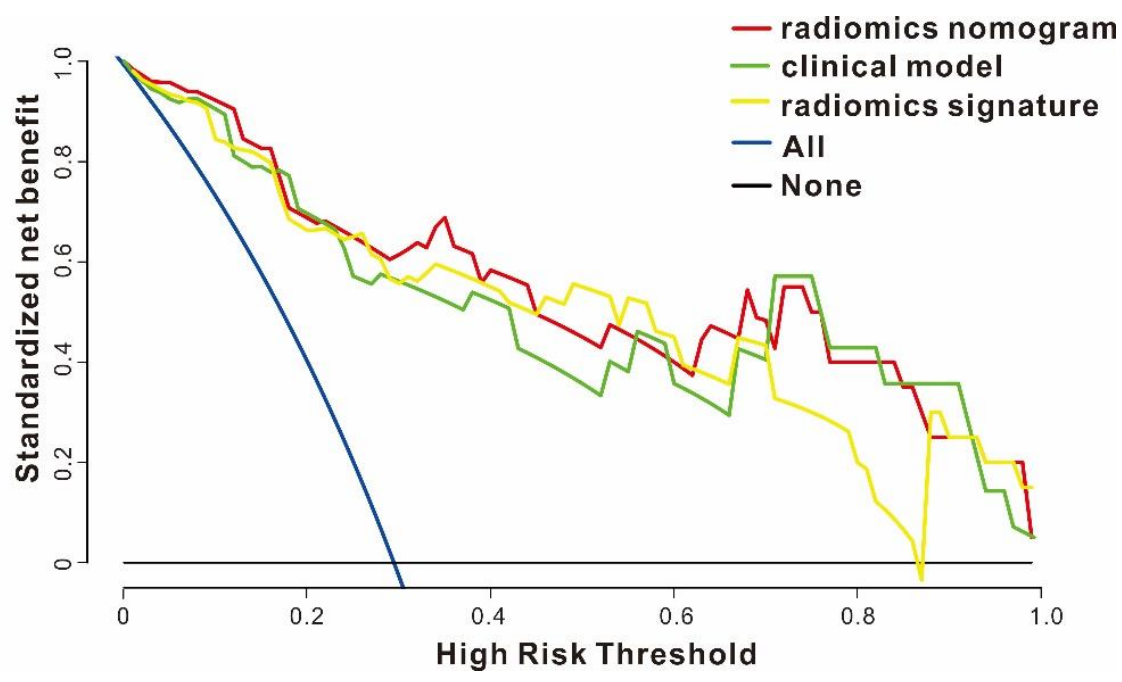

Fig.8 Decision curve analysis for three predictive models in predicting synchronous distant metastasis. The y-axis shows the net benefit. The x-axis shows the threshold probability. The blue line represents the assumption that all patients had metastasis. The black line represents the hypothesis that no patients had metastasis. 


\section{Supplementary Files}

This is a list of supplementary files associated with this preprint. Click to download.

- Supplementary.docx 